All autosomal variants were filtered for allelic frequency $<1 \%$ according to the gnomAD database (version 2.1). Among them, 821 variants were found the most common $(\geq 10 \%)$ in both the control and experimental group. Wherein 280 variants were presented with an alternative allele frequency of more than $10 \%$ in the experimental group, but were found with a lower frequency in the control. Interestingly, 10 of these 280 variants were located in the ZNF717 gene. This gene encodes a Kruppel-associated box (KRAB) zinc-finger protein, which belongs to a large group of transcriptional regulators in mammals and play important roles in various cellular functions, including cell proliferation, differentiation and apoptosis. Extended bioinformatics analysis showed that 34 unique variants were found in the experimental group and were absent in the control group, which may indicate both the characteristics of Russian children with BPD and the insufficient representativeness of the control group and, in turn, requires a more in-depth analysis.

For the first time in Russia, large-scale studies have been carried out to identify the molecular genetic characteristics of Russian children with BPD using full exome sequencing. Our study indicates ZNF717 gene may be relevant in BPD pathogenesis, but further research is required. These preliminary results may contribute to improving knowledge of the pathogenesis of bronchopulmonary dysplasia and targeting therapeutic interventions.

\section{WHAT IS BEHIND A SPONTANEOUS PNEUMOTHORAX?}

Joana Filipe Ribeiro*, Iris Santos Silva, João Virtuoso, Pedro Fernandes, Glória Silva, Rita S Oliveira, Pedro Carvalho. Department of Pediatrics, Hospital Sousa Martins, ULS Guarda

10.1136/archdischild-2021-europaediatrics.424

Introduction/Description Secondary Spontaneous Pneumothorax has its peak incidence in adulthood, occurring as a complication of an underlying lung disease, with variable symptoms at presentation.

A 15-year-old female previously healthy was admitted to the Emergency Department complaining of chest pain on left hemithorax and dyspnoea lasting for 24 hours, associated with a non-productive cough for 1 week. She denied fever, rhinorrhea, trauma, foreign body aspiration, inhalation of drugs, consumption of tobacco or any medication. She had no relevant family history. On admission, she was eupneic, SpO2 $99 \%(\mathrm{FiO} 2$ 21\%) and an absence of vesicular murmur was noted on the left hemithorax. Chest radiography revealed a left side pneumothorax with no mediastinal deviation.

Supplemental oxygen was initiated by a nonrebreathing facemask and the patient was advice to rest. The lack of improvement motivated a pneumothorax drainage by needle aspiration with an initial favourable response. However, 24 hours later a recurrence of the pneumothorax was noted in the control radiography. Chest-CT confirmed this finding and depicted an atelectasis of the left lower lobe, with a slight right deviation of the upper mediastinum and left apical pulmonary cystic features, suggestive of Congenital Cystic Adenomatoid Malformation (CCAM) of the lung. Subsequently, surgical removal of the left upper lobe was performed and CCAM type II was confirmed by histopathology.

Conclusion Although primary spontaneous pneumothorax in adolescents is the most common subtype, in cases where the course of the disease is not as expected, a secondary cause should be considered.

Even though CCAM is considered a rare disorder, it is the most common congenital pulmonary malformation with an incidence of $1: 8300$ to 35.000 live-births. CCAM occurs mostly sporadically and in about $1 / 3$ of the cases the diagnosis is made after the neonatal period. In the presence of pneumothorax, CCAM type IV should be considered and deserves special attention due to its malignant potential. In symptomatic cases, the therapeutic measures consist in pulmonary resection of the affected areas, which is curative and has few complications.

\section{A CASE OF A 13-YEAR-OLD BOY WITH PARAPNEUMONIC EFFUSION CAUSED BY STREPTOCOCCUS CONSTELLATUS}

Charisti Zampetaki*, Ioanna Farakla, Eufrosini Mpriasouli, Theoni Petropoulou. First Department of Paediatrics, National and Kapodistrean University of Athens, Greece, Aghia Sophia Children's Hospital

\subsection{6/archdischild-2021-europaediatrics.425}

A 13-year-old previously healthy male presented with a twoweek history of intermittent fever and left subcostal pain, which had previously been attributed to viral gastroenteritis. He was tachypnoeic, in need of supplemental oxygen, and on examination there was no air entry on the left side of the chest. Laboratory findings included leucocytosis (WBC 27000/ $\mu \mathrm{l}$ with a left shift) and high levels of inflammatory markers (CRP>300 mg/L).

Chest X-ray revealed complete whiteout of the left lung and subsequent ultrasound showed a large loculated pleural effusion. CT chest confirmed the findings and showed the extent of the infection and effusion. The patient was taken to theatre where a chest drain was inserted, automatically draining approximately $500 \mathrm{ml}$ of pus-like fluid, which was sent for cell count, microscopy, and cultures.

The patient was started on broad-spectrum intravenous antibiotics and received intrapleural fibrinolysis treatment with alteplase. His PCR and antibodies for SARS-CoV2 were negative. The pleural fluid analysis confirmed an exudate, and molecular methods as well as pleural fluid cultures indicated Streptococcus constellatus as a cause for his pneumonia and subsequent complicated parapneumonic effusion. Sputum cultures and blood cultures were negative.

Despite optimal antibiotic treatment with cefotaxime and teicoplanin based on sensitivities, there was little clinical and radiological improvement over the next few days, therefore the decision was made for the patient to return to theatre for decortication of the left lung and thoracostomy.

Following this steady progress was made and the patient was able to mobilize and benefit from physiotherapy to help expand the left lung.

Inflammatory markers returned to normal, and imaging showed marked improvement. The patient completed a total of 3 weeks of intravenous antibiotics. The chest drain was removed on day 18 of his hospital stay after having drained a total of over $1500 \mathrm{ml}$ of fluid.

Prior to discharge, as the streptococcus species isolated was not considered typical of the patient's age group and course of illness further tests were sent. Streptococcus constellatus is a group $\mathrm{F}$ beta streptococcus species, known to cause high 